\title{
Secondary pigmentary glaucoma in patients with underlying primary pigment dispersion syndrome
}

This article was published in the following Dove Press journal:

Clinical Ophthalmology

20 March 2013

Number of times this article has been viewed

\author{
Kavitha R Sivaraman \\ Chirag G Patel \\ Thasarat S Vajaranant \\ Ahmad A Aref \\ Department of Ophthalmology and \\ Visual Sciences, Illinois Eye and Ear \\ Infirmary, University of Illinois at \\ Chicago School of Medicine, \\ Chicago, IL, USA
}

\begin{abstract}
Primary pigment dispersion syndrome (PPDS) is a bilateral condition that occurs in anatomically predisposed individuals. PPDS may evolve into pigmentary glaucoma, but it is difficult to predict which patients will progress. Secondary pigment dispersion is more often unilateral and acquired as a result of surgery, trauma, or intraocular tumor, but can likewise lead to pigmentary glaucoma. We report two cases of patients with bilateral PPDS who developed secondary pigment dispersion and pigmentary glaucoma in one eye. Patients with PPDS who acquire a secondary mechanism of pigment dispersion may be at an increased risk of progression to pigmentary glaucoma, presumably due to an increased burden of liberated pigment. In addition to regular surveillance for progression to glaucoma from PPDS, secondary causes of pigmentary dispersion in these eyes should be considered when patients present with grossly asymmetric findings. When secondary pigment dispersion is identified in eyes with PPDS, we recommend prompt intervention to alleviate the cause of secondary pigment dispersion and/or aggressive control of intraocular pressure to limit glaucomatous damage.
\end{abstract}

Keywords: primary pigment dispersion syndrome, pigmentary glaucoma

\section{Introduction}

Primary pigment dispersion syndrome (PPDS) is a clinical entity defined by excessive pigment liberation from the iris. PPDS is typically bilateral and affects young, myopic males. Classic anterior segment findings include pigment deposition on the corneal endothelium (Krukenberg spindle), dense pigmentation of the trabecular meshwork (TM), and mid-peripheral iris transillumination defects. ${ }^{1,2}$ A characteristic concave iris configuration has also been described, which is thought to facilitate pigment release through increased iridozonular contact. ${ }^{3,4}$ Over time, chronic pigment release can lead to elevated intraocular pressure and frank pigmentary glaucoma. The rate of progression to pigmentary glaucoma among patients with PPDS has been reported at $10 \%-50 \%,{ }^{2,5-8}$ with a prospective trial showing that the intraocular pressure (IOP) at presentation is a key risk factor for progression. ${ }^{8}$

Unlike PPDS, secondary pigment dispersion typically occurs in patients without any anatomic or structural predisposition. There is no age or gender predilection and the disease is often unilateral. Mechanisms of secondary pigment dispersion include iris chaffing from an intraocular lens (IOL), pigment shedding from an intraocular melanoma or other neoplasm, and chronic mydriatic therapy. ${ }^{9-11}$ Secondary pigment dispersion therefore encompasses a variety of pathologies that share a final common pathway of excessive pigment liberation. Regardless of the underlying cause, patients with secondary pigment dispersion may also progress to pigmentary glaucoma. 
Although PPDS and secondary pigment dispersion have previously been construed as two distinct entities, we present two cases that demonstrate the development of secondary pigment dispersion and pigmentary glaucoma in patients with underlying PPDS. To our knowledge, the coexistence of secondary pigment dispersion in an eye with PPDS has not previously been reported. In light of the significant visual morbidity posed by pigmentary glaucoma, we believe it is important to alert treating ophthalmologists that when secondary pigment dispersion occurs in eyes with PPDS, it may hasten progression to pigmentary glaucoma.

\section{Description of cases Case I}

A 54-year-old Caucasian male presented to our institution as a new patient. His past ocular history was significant for high myopia and routine cataract extraction with IOL implantation in the right eye at age 46. The IOL became dislocated after the patient sustained a fall at age 53, and he subsequently underwent placement of a scleral-fixated three-piece IOL. The patient presented to our office approximately 1 year after this procedure and complained only of chronically decreased vision bilaterally. Best-corrected visual acuity was 20/60 in the right eye and 20/100 in the unoperated left eye. IOP was $46 \mathrm{mmHg}$ in the right eye and $17 \mathrm{mmHg}$ in the left. Slit-lamp examination was significant for bilateral Krukenberg spindles, but did not reveal any corneal edema. Gonioscopy showed 3+ homogenous pigmentation of the TM in both eyes by the Spaeth grading system with otherwise open angles and a markedly concave iris configuration bilaterally. No angle recession was noted. The right eye demonstrated a quiet anterior chamber with marked iridodonesis, but no pseudophacodonesis. The right eye also showed patchy transillumination defects throughout the iris stroma with intermittent pupil capture of the IOL optic (Figure 1A-C). The left iris showed mid-peripheral transillumination defects in the superotemporal quadrant. Ultrasound biomicroscopy confirmed the concave iris configuration, as well as apposition of the iris with the IOL (Figure 1D). The left eye revealed a mature nuclear sclerotic cataract without phacodonesis.

The right optic nerve demonstrated advanced cupping and pallor compared to the left (Figure 2A and B). Automated Humphrey visual field testing showed marked generalized depression in the right eye and a relatively full field in the left (Figure 2C and D). IOP-lowering therapy was initiated in the right eye with topical brimonidine/timolol and travoprost, which reduced the IOP to $13 \mathrm{mmHg}$.

\section{Case 2}

Case 2 is a 55-year-old Caucasian male with no relevant past medical history who presented to our institution as an urgent referral with elevated IOP in the left eye. His past ocular history was significant for high myopia and an iris nevus of the left eye diagnosed at age 19. IOP at presentation was $19 \mathrm{mmHg}$ in the right eye and $50 \mathrm{mmHg}$ in the left. Slit-lamp exam of the right eye demonstrated a clear cornea, round

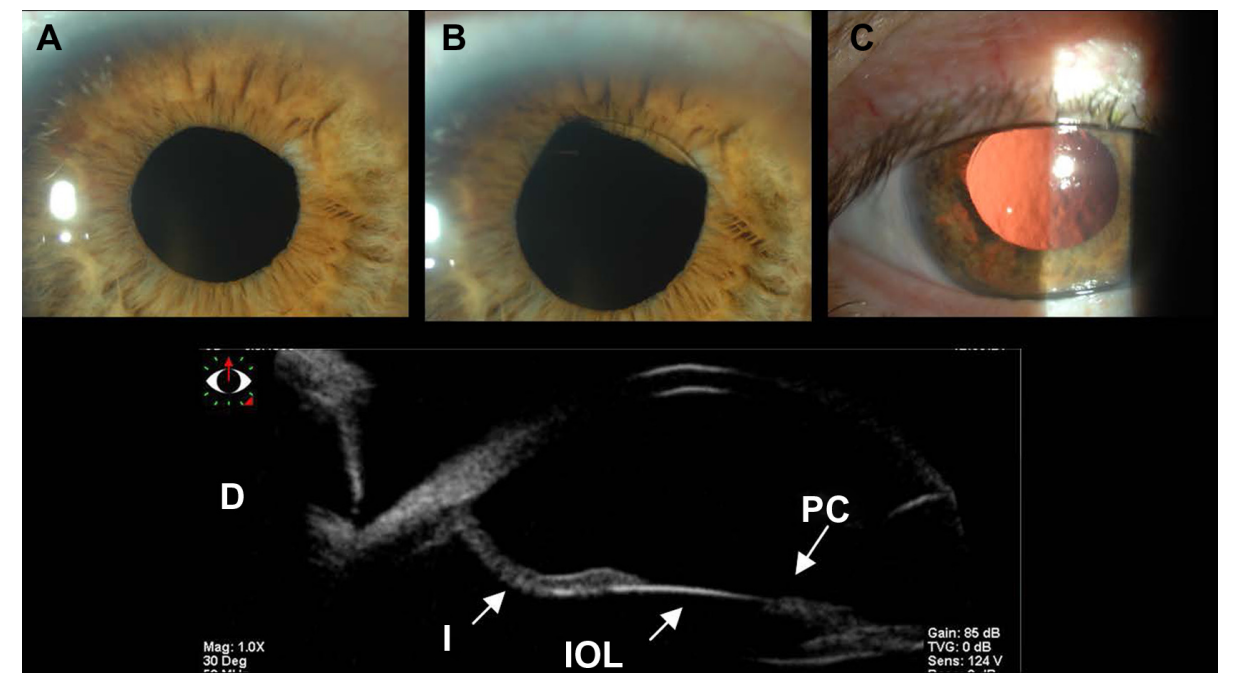

Figure I The right eye of case I demonstrates intermittent pupil capture of the IOL optic. The optic is initially behind the pupil (A), but becomes intermittently captured after a single blink (B). Postdilation slit-lamp photograph shows patchy transillumination defects with the superior IOL haptic and optic edge now visible (C). Ultrasound biomicroscopy (D) shows a concave I configuration and contact between the I and the IOL during PC.

Abbreviations: IOL, intraocular lens; I, iris; PC, pupil capture. 

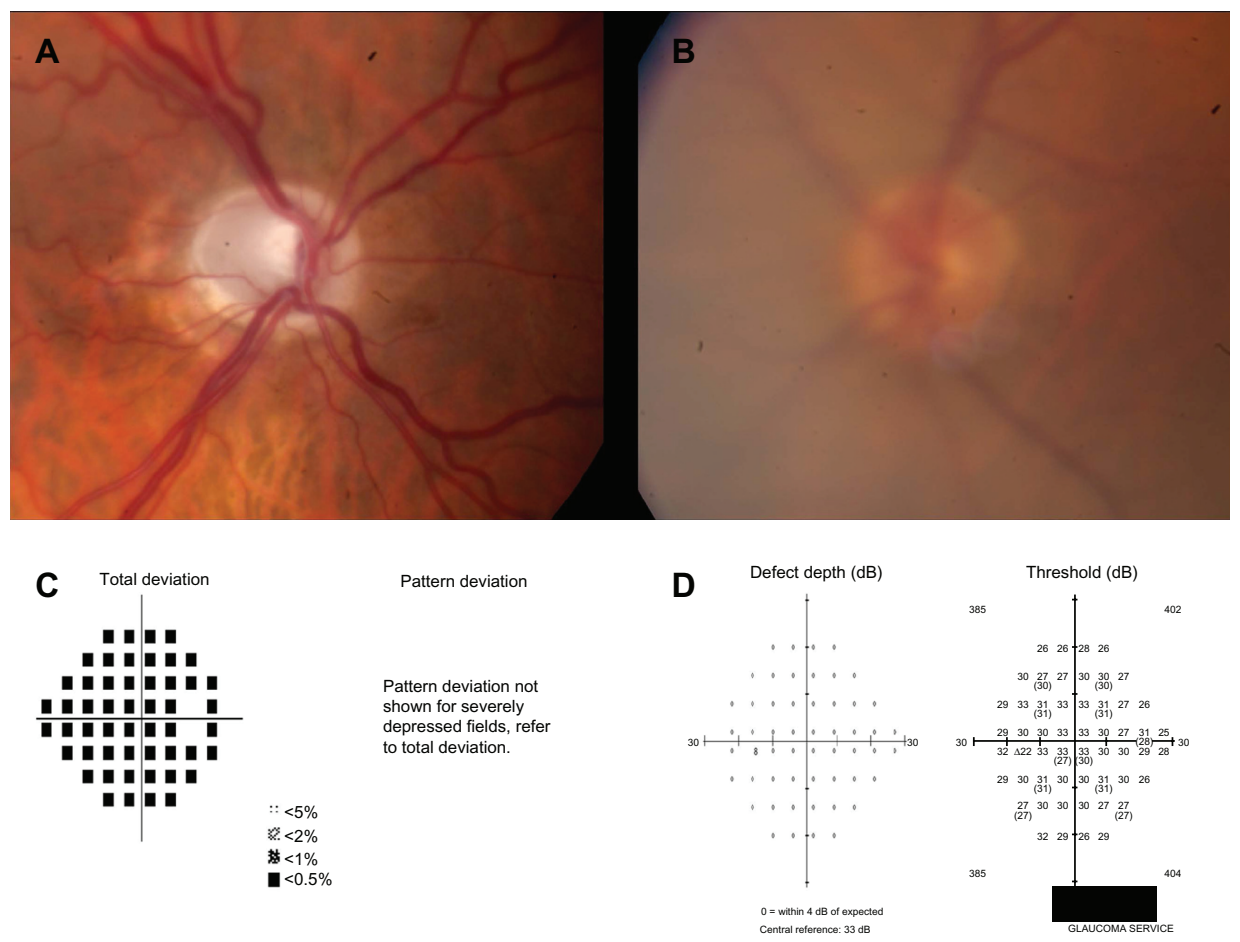

Figure 2 Optic nerve photographs. Optic nerve photographs of the right (A) and left (B) eyes of case I demonstrate advanced cupping and pallor of the right eye with a healthy neuroretinal rim in the left eye. Visualization of the left optic nerve is slightly impaired due to the presence of a mature cataract. Automated visual field testing of the right (C) and left (D) eyes shows marked generalized depression in the right eye.

Note: The left eye was tested with a size $V$ stimulus due to an advanced cataract, but shows a full field.

pupil without iris transillumination defects, a quiet anterior chamber, and pigment deposition on the posterior capsule of the crystalline lens (Scheie stripe; Figure 3). ${ }^{12}$ The left eye showed a clear cornea and deep anterior chamber with $2+$ pigmented cells (16-25 per high power field). The left eye also showed a darker overall iris color and an inferotemporal pigmented iris mass (Figure 4A). Gonioscopy revealed open angles in both eyes with $3+$ uniform pigmentation of the TM in the right eye by the Spaeth grading system. The left eye showed 4+ pigmentation in the superior, nasal, and temporal quadrants with extremely heavy pigmentation inferiorly (Figure 4B); both irides were noted to have a markedly concave configuration. The left optic nerve showed marked cupping compared to the right eye with a dense inferior arcuate scotoma on automated visual field testing (Figure 5A-D).

Ocular oncologic evaluation supported a benign iris nevus in the left eye based on ultrasound biomicroscopy appearance and transillumination. Additionally, serial photographs of the lesion failed to show any growth. Topical ocular antihypertensive therapy was initiated, but failed to achieve an adequately low IOP. The patient therefore underwent implantation of a glaucoma drainage implant with good postoperative IOP control.

\section{Discussion}

Sugar and Barbour ${ }^{1}$ first described pigmentary glaucoma in 1949 in their report of two patients with marked TM pigmentation, iris transillumination defects, and elevated IOP. These findings were later described to be secondary to a posterior bowing of the iris and increased pigment liberation from iridozonular contact. ${ }^{3,13}$ Secondary pigment dispersion can result from a variety of causes (trauma, surgery, intraocular tumor), as demonstrated in the cases above; however, the end result is still excessive pigment liberation that can lead to pigmentary glaucoma.

Case 1 illustrates a patient with Krukenberg spindles, heavy pigmentation of the TM, and a concave iris insertion consistent with bilateral PDS, but with glaucomatous damage and increased severity of pigment liberation in the eye with the scleral-fixated IOL. The patchy iris transillumination defects differ from the classic mid-peripheral spoke-like defects seen in classic PPDS, and thus suggest additional pigment chaffing from the IOL rubbing against the posteriorly bowed iris. The preservation of a concave iris insertion in patients with PPDS even after cataract surgery has been previously reported. ${ }^{14}$ Furthermore, ultrasound biomicroscopy imaging confirmed both apposition of the iris and IOL during pupillary capture (Figure 1D), and the absence of 


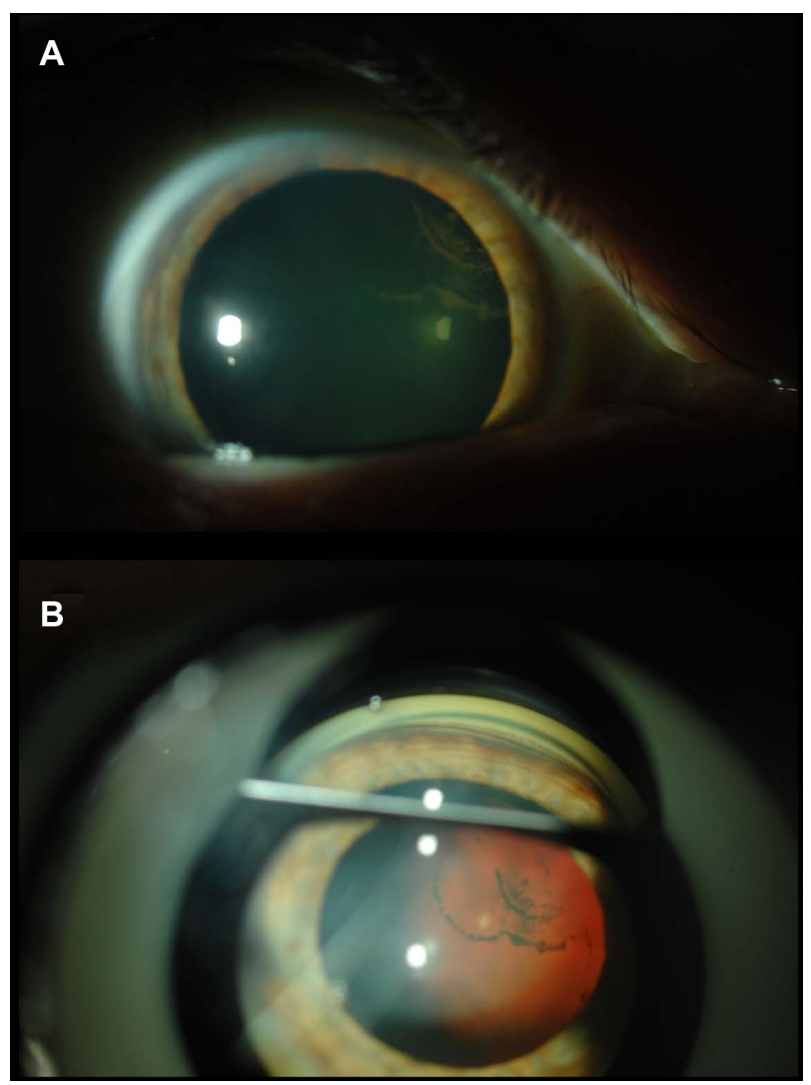

Figure 3 Postdilation slit-lamp photo of the right eye of case 2 shows posterior pigment deposition on the posterior lens capsule (Scheie stripe), consistent with a diagnosis of primary pigment dispersion syndrome $(\mathbf{A})$. Gonioscopy of the right eye shows an open angle with a concave iris configuration and 3+ uniform pigmentation of the trabecular meshwork (B).

pseudophakic pupillary block, as the angle is not occluded by peripheral iris. Additionally, as the patient was asymptomatic and the eye was quiet, the elevated IOP is more likely attributable to a chronic elevation, as seen in pigmentary glaucoma rather than an acute rise, as would be seen with a pseudophakic pupillary block. There were no inflammatory or red blood cells to suggest a uveitis-glaucoma-hyphema syndrome picture, which can also occur with IOLs placed in the ciliary sulcus.

The patient in case 2 had bilaterally increased, though asymmetric, pigmentation of the TM and concave iris insertions with a Scheie stripe in the right eye, again supporting the diagnosis of underlying PPDS. However, only the left eye developed frank pigmentary glaucoma. The source of presumed secondary pigment dispersion in this case was an iris nevus (Figure 4A); active pigment shedding was evidenced by the presence of pigmented cells in the anterior chamber on several visits, despite the lack of dilation or other provocative factors such as recent exercise. It is also notable that the patient was asymptomatic with a quiet left eye despite an IOP

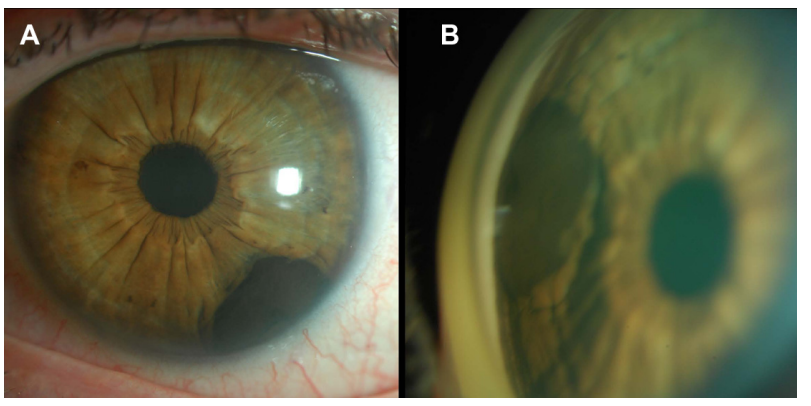

Figure 4 Slit-lamp photo and gonioscopy of the left eye. Slit lamp photo of the left eye of case 2 shows a darkly pigmented $4.2 \mathrm{~mm} \times 2.2 \mathrm{~mm}$ inferotemporal iris nevus (A). Gonioscopy of the left eye shows 4+ pigmentation of the trabecular meshwork (B).

of $50 \mathrm{mmHg}$, again suggesting a more gradual elevation in IOP, as may be seen in chronic pigmentary glaucoma.

The mechanism of chronic IOP elevation despite anatomically open angles in patients with PPDS is poorly understood, although IOP spikes after dilation or exercise are well-described. ${ }^{9}$ Histologic studies have shown that while trabecular endothelial cells have a substantial capacity for clearing pigment granules via phagocytosis, ${ }^{15}$ eyes with PPDS sustain trabecular cell loss and deposition of extracellular material under the inner wall of Schlemm's canal with obliteration of the canal itself. These pathologic changes are more severe in eyes with frank pigmentary glaucoma, and are likely associated with a more chronic elevation in IOP. ${ }^{2,15}$

Although both primary and secondary pigmentary dispersion can lead to elevated IOP and the development of pigmentary glaucoma, the majority of patients still do not progress. ${ }^{2,5-8}$ Richardson proposed that progression from pigmentary dispersion to pigmentary glaucoma occurred in two distinct stages. ${ }^{2,16,17}$ In the first, transient IOP elevations occur in response to acute obstruction of the TM by pigment granules. ${ }^{2,16,17}$ In this phase the damage is reversible, but over time Richardson purports that the TM endothelial cells become permanently altered by the ongoing pigment phagocytosis and separate from the connective tissue lamellae of the TM., ${ }^{2,16,17}$ The trabecular beams are then left bare and eventually degenerate, leading to increased outflow obstruction and the second, irreversible phase of pigmentary glaucoma. ${ }^{15-17}$ This proposed pathogenesis suggests that both the chronicity and severity of pigment dispersion contribute to the pathologic changes in the TM. The TM endothelium of an eye with PPDS already has a substantial burden of pigment to clear; as demonstrated in the above cases, the addition of another source of pigment dispersion may accelerate disease progression towards critical TM compromise and frank pigmentary glaucoma. 


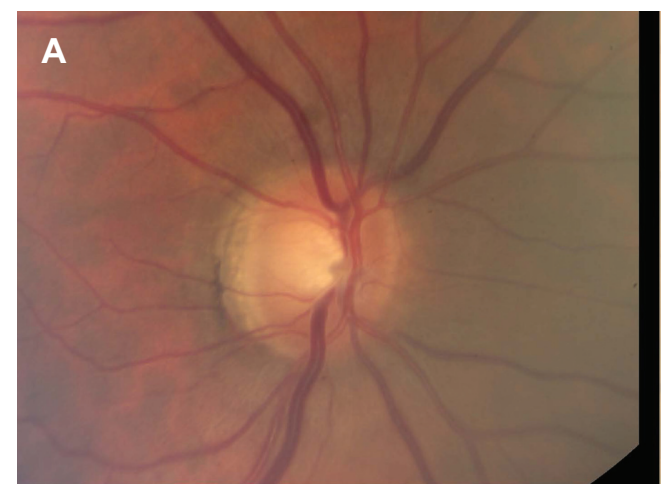

$B$
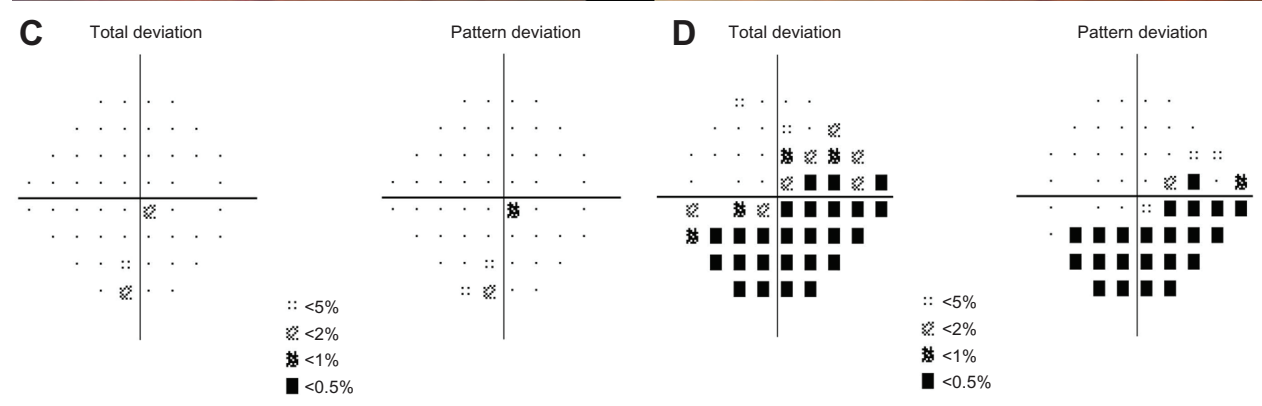

Figure 5 Optic nerve photographs of the right and left eyes of case 2. Optic nerve photographs of the right (A) and left (B) eyes of case 2 demonstrate a healthy neuroretinal rim in the right eye and advanced cupping with thinning of the inferior and superior neuroretinal rim in the left eye. Automated visual field testing of the right (C) and left (D) eyes revealed an unremarkable field in the right eye and a dense inferior arcuate scotoma in the left eye on pattern standard deviation.

It remains unclear if patients with PPDS are somehow more prone to developing secondary pigment dispersion, but it is possible that they are more sensitive to the effects of increased pigment liberation due to an underlying compromised TM. ${ }^{17}$ Indeed, it is well-described that IOP spikes after routine laser peripheral iridotomy occur more commonly in patients with PPDS, perhaps due to an increased tendency for the iris to release pigment. ${ }^{18}$ However, some proportion of this IOP rise could conceivably be due to an increased pigment load on an already stressed TM endothelium. The concentration of pigment granules in the anterior chamber has also been shown to correlate with maximum IOP and visual field loss in eyes with PPDS. ${ }^{19}$ Although we did not quantify the pigment granules in the cases described above, it is likely that eyes with underlying PPDS with an acquired secondary mechanism of pigment dispersion have a higher pigment burden than eyes with PPDS alone. This is exemplified by the extensive iris atrophy described in case 1, and the presence of active pigmented cells in the anterior chamber in case 2 .

Prior studies of the natural history of pigmentary glaucoma have shown a tendency for the disease to "burn out" with age. ${ }^{3,9}$ This is thought to be due to an increase in physiologic pupillary block, as the crystalline lens increases in its anterior-posterior diameter and displaces the peripheral iris forward and away from the zonules. ${ }^{3,9}$ There is also a decrease in accommodation with age that again may decrease iridozonular contact. However, the concept of a "burnt out" pigmentary glaucoma assumes that all of the pigment liberation is due to iridozonular contact alone, which is not the case in patients with secondary pigment dispersion, either in isolation or superimposed on PPDS. For example, despite age-related lens changes and a presumed decrease in accommodative tone, the patient in case 2 had ongoing pigment dispersion due to pigment shedding from an iris nevus. This underscores the importance of early intervention to either alleviate the cause of the secondary pigment dispersion or to aggressively control IOP.

In the two cases described above, patients with evidence of bilateral PPDS developed a secondary mechanism of pigment dispersion in one eye that went on to develop pigmentary glaucoma in an accelerated fashion. Since both patients presented to us after they had already developed pigmentary glaucoma in one eye, it is impossible to be sure that they did not have early glaucomatous optic neuropathy prior to developing secondary pigment dispersion. However, given the drastic asymmetry in both of these patients, it seems likely, if not probable, that the secondary pigment dispersion accelerated the course of their disease.

While the above cases still do not help predict which patients with PPDS will develop pigmentary glaucoma, we believe any patient with PPDS who acquires another 
mechanism for increased pigment dispersion may be at a higher risk and should be monitored very closely for the development of pigmentary glaucoma.

\section{Conclusion}

Acquired secondary pigment dispersion in an eye with PPDS has not previously been described. The cases above illustrate that eyes with PPDS and superimposed secondary pigment dispersion may be at an increased risk for progression to pigmentary glaucoma, perhaps due to a further increase in pigment liberation and damage to the TM. When this particular clinical scenario is identified, we advise prompt action to either treat the cause of the secondary pigment dispersion or to achieve aggressive control of elevated IOP in order to prevent glaucoma progression.

\section{Disclosure}

The authors report no conflicts of interest in this work.

\section{References}

1. Sugar HS, Barbour FA. Pigmentary glaucoma; a rare clinical entity. Am J Ophthalmol. 1949;32(1):90-92.

2. Scott A, Kotecha A, Bunce C, et al. YAG laser peripheral iridotomy for the prevention of primary pigment dispersion glaucoma a prospective, randomized, controlled trial. Ophthalmology. 2011;118(3):468-473.

3. Campbell DG. Pigmentary dispersion and glaucoma. A new theory. Arch Ophthalmol. 1979;97(9):1667-1672.

4. Liu L, Ong EL, Crowston J. The concave iris in pigment dispersion syndrome. Ophthalmology. 2011;118(1):66-70.

5. Richter CU, Richardson TM, Grant WM. Pigmentary dispersion syndrome and pigmentary glaucoma. A prospective study of natural history. Arch Ophthalmol. 1986;104(2):211-215.

6. Farrar SM, Shields MB, Miller KN, Stoup CM. Risk factors for the development and severity of glaucoma in the pigment dispersion syndrome. Am J Ophthalmol. 1989;108(3):223-229.
7. Migliazzo CV, Shaffer RN, Nykin R, Magee S. Long-term analysis of pigmentary dispersion syndrome and pigmentary glaucoma. Ophthalmology. 1986;93(12):1528-1536.

8. Siddiqui Y, Ten Hulzen RD, Cameron JD, Hodge DO, Johnson DH. What is the risk of developing pigmentary glaucoma from pigment dispersion syndrome? Am J Ophthalmol. 2003;135(6):794-799.

9. Niyadurupola N, Broadway DC. Pigment dispersion syndrome and pigmentary glaucoma - a major review. Clin Experiment Ophthalmol. 2008;36(9):868-882.

10. Hadid O, Megaw R, Owen R, Fraser S. Secondary pigment dispersion syndrome with single-piece acrylic IOL. J Cataract Refract Surg. 2010;36(9):1610-1611.

11. Shields CL, Shields JA, Shields MB, Augsberger JJ. Prevalence and mechanisms of secondary intraocular pressure elevation in eyes with intraocular tumors. Ophthalmology. 1987;94(7):839-846.

12. Scheie HG, Fleischhauer HW. Idiopathic atrophy of the epithelial layers of the iris and ciliary body; a clinical study. Trans Am Ophthalmol Soc. 1957-1958;55:369-388.

13. Karickhoff JR. Pigmentary dispersion syndrome and pigmentary glaucoma: a new mechanism concept, a new treatment, and a new technique. Ophthalmic Surg. 1992;23(4):269-277.

14. Moreno-Montañés J, Salinas-Alamán A, Barrio-Barrio J, Bonet-Farriol E. Pupil capture of intraocular lens in vitrectomized eye with primary pigment dispersion syndrome. $J$ Cataract Refract Surg. 2011;37(3):612-613.

15. Gottanka J, Johnson DH, Grehn F, Lütjen-Drecoll E. Histologic findings in pigment dispersion syndrome and pigmentary glaucoma. J Glaucoma. 2006;15(2):142-151.

16. Richardson TM, Hutchinson BT, Grant WM. The outflow tract in pigmentary glaucoma: a light and electron microscopic study. Arch Ophthalmol. 1977;95(6):1015-1025.

17. Richardson TM. Pigmentary glaucoma. In: Ritch R, Shields MB, editors. The Secondary Glaucomas. St Louis, MO: Mosby; 1982:84-98.

18. Birt CM. Intraocular pressure spike after YAG iridotomy in patients with pigment dispersion. Can J Ophthalmol. 2004;39(3):234-239.

19. Mardin CY, Küchle M, Nguyen NX, Martus P, Naumann GO. Quantification of aqueous melanin granules, intraocular pressure and glaucomatous damage in primary pigment dispersion syndrome. Ophthalmology. 2000;107(3):435-440.

\section{Clinical Ophthalmology}

\section{Publish your work in this journal}

Clinical Ophthalmology is an international, peer-reviewed journal covering all subspecialties within ophthalmology. Key topics include: Optometry; Visual science; Pharmacology and drug therapy in eye diseases; Basic Sciences; Primary and Secondary eye care; Patient Safety and Quality of Care Improvements. This journal is indexed on Submit your manuscript here: http://www.dovepress.com/clinical-ophthalmology-journal

\section{Dovepress}

PubMed Central and CAS, and is the official journal of The Society of Clinical Ophthalmology (SCO). The manuscript management system is completely online and includes a very quick and fair peer-review system, which is all easy to use. Visit http://www.dovepress.com/ testimonials.php to read real quotes from published authors. 